\title{
Safe performance of echocardiography during the COVID-19 pandemic: a practical guide
}

Matteo Cameli ${ }^{1, *}$, Maria Concetta Pastore ${ }^{1}$, Michael Henein ${ }^{2,3,4}$, Hatem Soliman Aboumarie ${ }^{5}$, Giulia Elena Mandoli ${ }^{1}$, Flavio D'Ascenzi ${ }^{1}$, Paolo Cameli ${ }^{6}$, Federico Franchi ${ }^{7}$, Sergio Mondillo ${ }^{1}$ and Serafina Valente ${ }^{1}$

\author{
${ }^{1}$ Department of Medical Biotechnologies, Division of Cardiology, University of Siena, Siena 53100, Italy \\ ${ }^{2}$ Department of Public Health and Clinical Medicine, Umeå University, Umeå 90187, Sweden \\ ${ }^{3}$ Department of Public Health and Clinical Medicine, St George London university, London SW17 ORE, United Kingdom \\ ${ }^{4}$ Department of Public Health and Clinical Medicine, Brunel University, Uxbridge UB8 3PH, United Kingdom \\ ${ }^{5}$ Adult Intensive Care Unit, Royal Brompton and Harefield NHS Foundation Trust, London SW3 6NJ, United Kingdom \\ ${ }^{6}$ Department of Clinical Medical and Neurosciences, Respiratory Disease and Lung Transplantation Section, Le Scotte \\ Hospital, University of Siena, Siena 53100, Italy \\ ${ }^{7}$ Department of Medical Biotechnologies, Anesthesia and Intensive Care, University of Siena, Siena 53100 , Italy \\ ${ }^{*}$ Correspondence: matteo.cameli@yahoo.com (Matteo Cameli)
}

DOI: $10.31083 /$ i.rcm.2020.02.90

This is an open access article under the CC BY 4.0 license (https://creativecommons.org/licenses/by/4.0/).

Coronavirus disease-2019 (COVID-19) outbreak has become a worldwide healthcare emergency, with continuously growing number of infected subjects. Considering the easy virus spread through respiratory droplets produced with cough, sneezes or spit or through close contact with infected people or surfaces, healthcare workers are further exposed to COVID-19. Particularly, echocardiography remains an essential diagnostic service which, due to the close contact with patients during the exam, provides echocardiographers high-risk of contagion. Therefore, the common modalities of performing echocardiography should be improved in this scenario, avoiding performing unnecessary exams, using the appropriate personal protective equipment depending on patients' status and location, optimizing time-effectiveness of the echocardiographic study and accurately sanitizing the environment and devices after each exam. This paper aims to provide a simple guide for the clinicians to balance between providing the best care to each patient and protecting themselves and other patients from the spread of the virus. It also proposes the use of the mnemonic PREVENT to resume the crucial indications to be followed for the execution of appropriate echocardiographic examination during the COVID-19 pandemic.

\section{Keywords}

COVID-19; echocardiography; SARS-CoV2; personal protective equipment; coronavirus; pandemic

\section{Introduction}

Coronavirus disease-2019 (COVID-19) pandemic is a worldwide public health burden, being associated with high morbidity and mortality risks, especially for specific groups of popula- tion: elderly, chronically ill, immunocompromised and pregnant women (Huang et al., 2020). Moreover, it is affecting the global provision of healthcare services. Coronavirus spreads easily from symptomatic or asymptomatic patients through direct contact and respiratory droplets associating cough or sneezes (Wu and McGoogan, 2020). Echocardiography is a routinely requested investigation in patients with unexplained breathlessness, particularly those with underlying cardiac conditions. The procedure is usually performed through close contact with patients, hence resulting in high contagious atmosphere. Patients with suspected or confirmed COVID-19 could fall in the category of those requiring echocardiographic assessment, a clinical need that puts the cardiologist/echocardiographer at a significant risk of catching severe acute respiratory syndrome coronavirus-2 (SARS-CoV2) infection. Thus, it is of paramount importance to scrutinize the use of echocardiography during the current COVID-19 pandemic in an attempt to protect healthcare professionals, their relatives (Adams and Walls, 2020) and also other patients, both for their own health benefit as well as for controlling the spread of the disease (Conticini et al., 2020). In addition to rationalizing the use of such popular investigation, echocardiography should be provided using the appropriate personal protective equipment (PPE).

This document aims to assist clinicians in recognizing the priority indications for echocardiographic examination and optimum required precautions during the COVID-19 outbreak, proposing the use of the acronym "PREVENT" (Fig. 1) to easily remember the pivotal steps for a conscious use of echocardiography in this emergency scenario.

\section{Transthoracic echocardiography (TTE) \\ 2.1 Before the exam}

Transthoracic echocardiography (TTE) is commonly requested as a routine investigation in large cardiac centers and general 


\section{Echocardiographic exam}

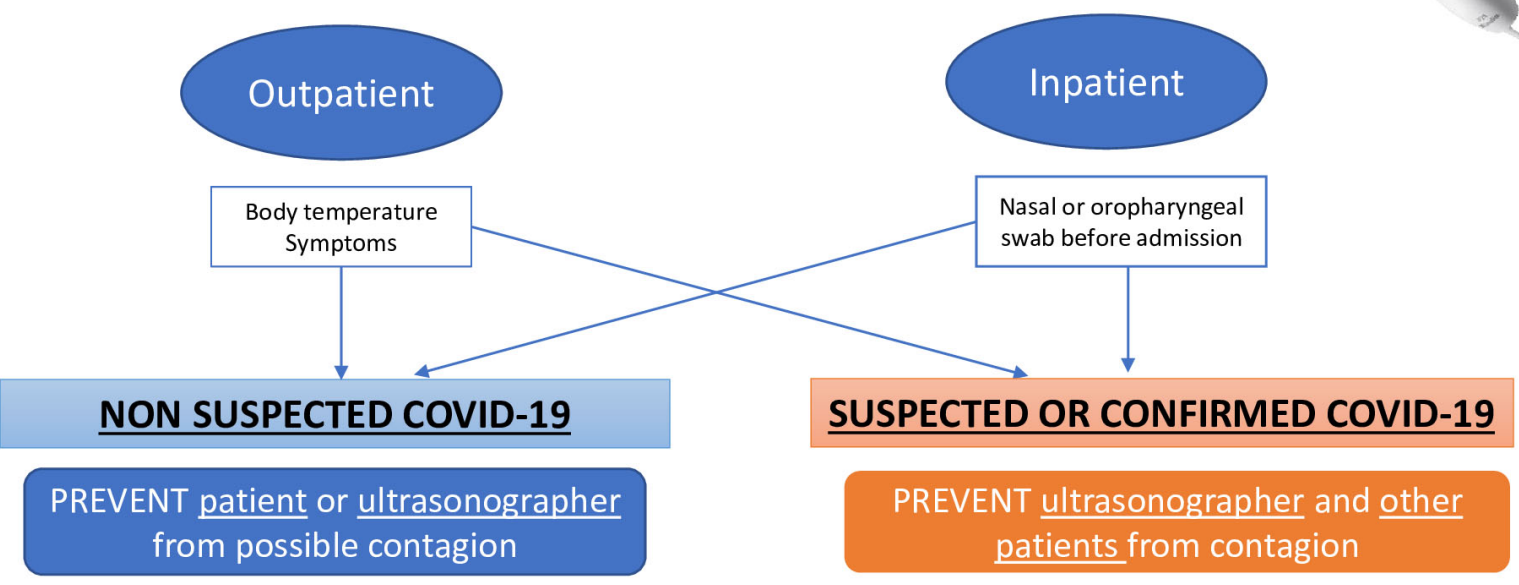

Patient COVID-19 status = non suspected $/$ negative Right indication? If NOT $\rightarrow$ RESCHEDULE EXAM

Equipment: standard precautions

Venue : Echo-labs or Hospital wards

Expedite : focused and planned TTE

Neatness : careful cleaning of device and surfaces

TOE: avoid if possible, or $\rightarrow$ droplets precautions

Patient COVID-19 status $=$ suspected $/$ positive Right indication? If NOT $\rightarrow$ RESCHEDULE EXAM

Equipment : droplets or airborne precautions

Venue: Hospital labs or wards, COVID Units, ICU

Expedite : focused and planned TTE

$N$ eatness : careful cleaning of device and surfaces

TOE: avoid if possible, or $\rightarrow$ airborne precautions

Figure 1. Indications for the appropriate use of echocardiography to PREVENT the spread of COVID-19. COVID-19, coronavirus disease-19; ICU, intensive care unit; TOE, transoesophageal echocardiography; TTE, transthoracic echocardiography

hospitals, where cardiac units of significant size, with enough echocardiographic apparatuses, provides routine daily service to other medical specialties. However, considering the high risk of catching COVID-19 from any patient, it is advisable that only necessary examinations should be undertaken, particularly in patients with unknown COVID status (Kirkpatrick et al., 2020). Patient COVID-19 status must be assessed according to local protocols. Screening for infection should be undertaken in outpatients' settings (body temperature measurement and the presence/absence of respiratory symptoms, i.e. cough, cold, flu, dyspnea over the prior 14 days) at the hospital/clinic entrance. As for hospitalization, all patients with suspected COVID-19 should undergo nasal and/or pharyngeal swab for the detection of SARS/CoV2/Nucleic Acid before admission.

Accordingly, the American Society of Echocardiography (ASE) and the European Association of Cardiovascular Imaging (EACVI) recommend that each elective echocardiogram should be postponed, regardless of patients' COVID status (non-suspected, suspected or confirmed infection) (Kirkpatrick et al., 2020; Skulstad et al., 2020). Nevertheless, primary cardiac symptoms and underlying cardiovascular disease should be recognized in a timely fashion, since they could often carry higher mortality than
COVID-19 itself. In general, Italian Society of Echocardiography and Cardiovascular Imaging (SIECVI) recommends cardiologists to preserve the chance to distinguish between urgent and undeferrable TTE exams and inappropriate requests at their personal judgement (Antonini-Canterin et al., 2020). However, as a reference guide, in Table 1 we suggest a list of the most common clinical conditions which could represent $\mathbf{R}$ ight indications to echocardiography during COVID-19 pandemic.

The key points for optimum management of TTE service during COVID-19 pandemic are:

i. An initial screening of the indication, following Table 1 or general appropriateness criteria for the use of TTE in adults (Chambers et al., 2017).

ii. TTE services should not be offered if unlikely to provide prompt clinical benefit to patients, which entails the prioritizing and guiding towards adequate short-term management ( $\mathrm{Wu}$ and McGoogan, 2020).

iii. Identifying urgency or emergency indications for TTE and deferring all others or define them as "elective" studies and reschedule them (Wu and McGoogan, 2020).

iv. Prefer alternative forms for distance-consulting, with network evaluation of echocardiographic images or telemedicine, 
Table 1. Appropriate and deferrable indications for echocardiography optimization during COVID-19 pandemic.

\begin{tabular}{ll}
\hline Appropriate indication & Deferrable \\
\hline Chronic HF (Pastore et al., 2019) with signs and $\begin{array}{l}\text { Chronic HF with absent or mild symptoms (Sciaccaluga et al., 2020) (NYHA } \\
\text { class I-II) }\end{array}$ \\
symptoms of congestion* \\
-Dyspnea \\
-Crackles \\
-Peripheral edema
\end{tabular}

Known mild-severe VHD or prosthetic valves with Routine echocardiographic evaluation in asymptomatic patients with underlying new symptoms cardiac disease

-Syncope

-Chest pain

-Dyspnea at rest

-New cardiac murmur (concomitant dental abscess or pain?)

Chest pain in previous CAD or known CCS, with Uncontrolled blood pressure levels $(<200 / 120 \mathrm{mmHg})$ in hypertensive patients negative troponin

High suspect of infective endocarditis

Cardiologic follow up of asymptomatic oncologic patients and without known or suspect CTRCD

Signs and symptoms of congestive acute $\mathrm{HF}^{*}$

Low-intermediate risk NSTEMI (for prioritization to STEMI or high risk NSTEMI (if patient is nearly going invasive coronary anPCI) giography) (Valente et al., 2020)

\begin{tabular}{|c|c|}
\hline Malignant arrhythmias & Dyspnea with low BNP blood levels \\
\hline $\begin{array}{l}\text { Pre-operative evaluation of patients referred for } \\
\text { high-risk surgery }\end{array}$ & Pre-operative evaluation of patients referred for intermediate or low-risk surgery \\
\hline Suspected STEMI complications & Study of symptomatic CCS if coronary CT is available (Skulstad et al., 2020) \\
\hline Hemodynamic instability & $\begin{array}{l}\text { Routine follow up of prosthetic valves in asymptomatic or with mild-symptoms } \\
\text { patients }\end{array}$ \\
\hline
\end{tabular}

ICU*-> suspected acute HF (Cameli et al., 2019; Lancellotti et al., 2015) TOE for diagnostic workup or elective surgical treatment of VHD High-PEEP ventilation (Franchi et al., 2013)

Veno-venous ECMO *Lung ultrasound could aid diagnosis (Gargani et al., 2020). BNP, brain natriuretic peptide; CAD, coronary artery disease; CCS, chronic coronary
syndromes; CT, computed tomography; CTRCD, chemotherapy-related cardiac disease; HF, heart failure; ICU, intensive care unit; NSTEMI, non-ST-
elevation myocardial infarction; NYHA, New York Heart Association; PCI, percutaneous coronary intervention; PEEP, positive end-expiratory pressure;
STEMI, ST-elevation myocardial infarction; TOE, transoesophageal echocardiography; VHD, valvular heart disease

in order to limit patient-operator contact (Driggin et al., 2020).

v. Repeated TTE should not be performed, except for clear changes of patients' clinical status.

vi. Cardiac specific blood biomarkers, such as brain natriuretic peptide (BNP) or N-terminal-proBNP and cardiac troponin, could be assessed to exclude acute cardiac injury or worsening chronic heart failure, in an attempt to reduce the number of unnecessary TTEs. However, the possible interference of SARS$\mathrm{CoV} 2$ with cardiac biomarkers should be considered, since it has shown to provoke an increase of troponin and BNP levels parallel to the worsening severity of the respiratory disease (Chen et al., 2020; Lippi et al., 2020).

\subsection{Performing the exam}

Given that the patient's indication to undergo rest or stress TTE is appropriate, the environment and the modalities of execution are crucial elements for overall personnel safety during the COVID-19 outbreak.

First of all, the operator should use the appropriate Equipment, according to local standards, which is recommended for the prevention of virus spread. Echocardiographers dealing with suspected or known COVID-19 patients may have different levels of personal protective equipment (PPE), depending on the Venue where TTE should be performed (Kirkpatrick et al., 2020):

i. Droplets precautions: gown, gloves, headcover, facemask (filtering face piece particulate class 2 (FFP2), class 3 (FFP3), N95 or N99 respirators masks) and eye shield for outpatients in echo-labs or inpatients in hospital wards or emergency department; patients should wear at least surgical mask as well.

ii. Airborne precautions: gown, gloves, headcover, eye shield, with special facemask (i.e. FFP2, FFP3, N95 or N99 respira- 
tors masks, or powered air purifying respirator (PAPR) systems) and shoe covers for patients hospitalized in intensive care units, or ventilated patients treated with invasive/noninvasive oxygen therapy.

In case of NON-suspected or known COVID-19 patient, TTE could regularly be performed in echo-labs or hospital wards, according to the new world health organization (WHO) recommendations, which states that only patients undergoing aerosolgenerating procedures require airborne isolation (World Health Organization, 2020a). Nevertheless, standard precautions are essential, i.e. frequent and meticulous handwashing, use of gloves and surgical masks; patients wearing surgical mask.

Given the above, it is fundamental to ensure the education of healthcare professionals on the correct methods of dressing/undressing. WHO (World Health Organization, 2020b) specific document reports detailed information about the rational use of PPE for COVID-19 prevention.

It is important to Expedite the duration of TTE examination, limiting its use to answer the clinical question, since prolonged scanning could expose the operator to increased risk of infection (Kirkpatrick et al., 2020; Skulstad et al., 2020). Therefore, echocardiographic exams in patients with suspected or confirmed COVID-19 should be:

i. focused to the indication question and prepared in advance based on findings of previous examination and cardiac condition details.

ii. on the other hand, TTE should be comprehensive enough to avoid the need for repeat examination or other investigations;

iii. performed by experienced operators (not students or intraining practitioners) in order to optimize the time/quality ratio of images acquisition;

iv. ultrasound enhancing agent (contrast) use should be planned in advance, so as to prevent the unnecessary waiting time or/and the need for personal potential contamination of other areas while preparing the contrast.

v. Advanced analyses or measures, such as speckle tracking echocardiography (Cameli et al., 2013), should not be overlooked if they could provide relevant additional information for the clinical question, however, they should be carefully performed offline in a safe environment, using TTE images acquired and saved in a storage driver.

Some authors suggest that portable echocardiographs could be used to perform TTE in suspected or known COVID-19 subjects in order to avoid SARS-CoV2 transmission throughout the hospital while moving them, and for their much easier cleaning procedures (Kirkpatrick et al., 2020; Skulstad et al., 2020; World Health Organization, 2020b). However, usually COVID-19 patients are isolated in dedicated units, and the capability of the virus to remain active on surfaces for several days should be considered (Kampf et al., 2020; van Doremalen et al., 2020). Hence, perhaps the best option is the use of a unique device in infected areas by a dedicated echocardiographer who follows optimum hygiene recommendations (Antonini-Canterin et al., 2020) and avoiding carrying unnecessary objects e.g. electrodes for ECG trace, additional probes.
Alternatively, special protocols known as "point-of-care ultrasound (POCUS)" (Kirkpatrick et al., 2020) or "FocUS" (Neskovic et al., 2018), performed by trained clinicians by handheld devices, can play a role in optimizing TTE evaluation at referral to the emergency department or for repeated evaluations in critical patients, to obtain a rapid assessment of biventricular function, valvular disease, pericardial and pleural effusion (Lisi et al., 2012). This approach could be useful to screen for cardiac contributions to symptoms or signs during COVID-19 infection or to identify patients who need complete echocardiographic examination, avoiding the exposure of other clinicians to SARS-CoV2 (Cameli et al., 2020). In doubtful cases, images obtained by POCUS can be archived to allow remote visualization or future review from more experienced echocardiographers for interpretative consulting or comparisons of cardiac structure and function over time.

\subsection{After the exam (Sanitize)}

Noteworthy, Neatness of the room and devices is crucial to prevent COVID-19 contagion. Therefore, after TTE exam, accurate sanitization of all the equipment is recommended (British Society of Echocardiography , 2020). SARS-CoV-2 has shown to be sensitive to most standard viricidal disinfectant solutions (van Doremalen et al., 2020). WHO suggested that accurate cleaning of the environmental surfaces with water and detergents, followed by the application of disinfectant commonly used within hospital (i.e. ethanol $62-71 \%$, sodium hypochlorite $0.1-0.5 \%$ or hydrogen peroxide $0.5 \%$ ) would be sufficient (Kampf et al., 2020).

According to the variety of local standards, it is important to ensure a thorough cleaning of echocardiograph and probes early after using them, while providing room ventilation. Wet swabs with 70\% alcoholic solution for the echocardiographs, and ammonium quaternarium for the probes, could be used (Skulstad et al., 2020). Nonetheless, cleaning procedures could also vary among vendors, thus it is advisable to adhere to individual vendors disinfecting guidelines.

Lastly, the staff participation should be limited to the ones performing TTE exam, avoiding people gatherings to review or discuss TTE findings. If necessary, remote images review and clinical consultation via telephone or web-networks are recommended.

\section{Transoesophageal echocardiography (TOE)}

TOE is characterized by very high risk of SARS-CoV-2 transmission, since it can cause aerosolization of a large amount of virus.

Therefore, the recommendations for TOE performance are quite different than those for TTE (Kirkpatrick et al., 2020):

i. TOE should be postponed or cancelled unless urgent, if it is unlikely to change clinical care, and/or if alternative TTE (e.g. off-axis TTE views, use of ultrasound enhancing agents) could be sufficient to obtain the necessary information.

ii. In patients with non-suspected COVID-19 infection, droplets (gown, gloves, headcover, eye shield, FFP2/FFP3 or N95/N99 respirators masks) precautions should be used.

iii. In patients with suspected or confirmed COVID-19, airborne precautions (gown, gloves, headcover, eye shield, FFP2/FFP3, N95/N99 or PAPR respirator masks) should be used 
whenever TOE is performed.

Obviously, further care than usual should be provided during the disinfection of the echocardiograph and the probe after TOE, according to vendors' specific guidelines.

\subsection{Use of echocardiography to study MIS-C}

Among the most worrisome complications of COVID-19, Multisystemic Inflammatory Syndrome in Children (MIS-C) has recently been discussed by pediatricians, rheumatologist and cardiologist communities (Sinha et al., 2020). This is probably caused by an acute severe cytokine storm and resembles toxic shock syndrome and atypical Kawasaki disease (KD). It frequently leads to acute heart failure and cardiogenic shock often representing the first manifestation of COVID-19 in children and adolescent, requiring inotropes and cardiac mechanical assistance (Belhadjer et al., 2020). To date, more than 200 patients with MIS-C with temporal association with COVID-19 have been reported.

According to the newest studies on patients with MIS-C, electrocardiograms could be non-specific, while echocardiography show the same imaging findings of patients with $\mathrm{KD}$ (McCrindle and Cifra, 2017; Riphagen et al., 2020; Verdoni et al., 2020). Therefore, we hypothesize that TTE would be useful not only for diagnosis of MIS-C, but also for prognostic assessment and follow up in case of coronary aneurysms, similarly to KD (Belhadjer et al., 2020).

The focusing points of a TTE study in suspected MIS-C are the following:

- Study of LV regional and global function, calculation of ejection fraction (often reduced, with possible recovery after intravenous immunoglobulin and steroids therapy);

- Dedicated views for the study of coronary arteries (McCrindle and Cifra, 2017) to assess dilatation (with Z-score $>2$ (Sosa et al., 2019) based on body surface area) or echo-brightness;

- Evaluation of pericardial effusion;

- Detection of unknown valvular regurgitation (above all mitral regurgitation).

TTE is the first line imaging method to use in patients with MIS-C. In patients with coronary aneurysms, the risk assessment for myocardial ischemia becomes pivotal. Serial exercise (preferable) or pharmacological stress echocardiography could provide further risk stratification (sensitivity $94.4 \%$ and specificity $82.5 \%$ at beginning, $93.7 \%$ and $95.4 \%$ respectively at 15 -years follow up) (Noto et al., 2013). Non-invasive imaging methods are recommended as alternatives to coronary angiograms. Coronary computed tomography scan may have a role for its high anatomic resolution and accuracy in identifying coronary stenosis and thrombi, however, it should be limited to high-risk cases due to radiation exposure; cardiac magnetic resonance would be useful to avoid radiations, but has the pitfalls to be time-consuming and less available (Tsuda and Singhal, 2017). Moreover, in COVID-19 times the use of these imaging methods should be optimized in favor of the protection of healthcare personnel and hospital environment.

\section{Conclusions}

COVID-19 outbreak exposes healthcare professionals to high infective risk with subsequent high morbidity and mortality rates, hence there is the need to reconsider the common indications and best appropriate modalities for optimum service. An optimization of TTE performance and the careful use of PPE is required, in order to ensure the adequate protection to healthcare professionals, other patients and overall surrounding environment, without compromising healthcare services provision. Following the recent evidence about COVID-19 and the recommendations of international societies of echocardiography, this document provides a set of basic suggestions to observe when performing TTE at the time of COVID-19 pandemic. Accordingly, we propose the clinicians to bear in mind the acronym PREVENT, which resumes the key indications to perform a safer echocardiographic exam, reducing the risk of COVID-19 contagion (Fig. 1).

\section{Authors' contributions}

All authors contributed to this review conception and draft of the manuscript, critically revised the work, and approved the final manuscript.

\section{Acknowledgements}

The authors declare no funding, no contribution from other persons.

\section{Conflict of interest}

The authors declare no conflict of interest.

Submitted: May 13, 2020

Revised: June 15, 2020

Accepted: June 18, 2020

Published: June 30, 2020

\section{References}

Adams, J. G. and Walls, R. M. (2020) Supporting the Health Care Workforce During the COVID-19 Global Epidemic. JAMA 323, 1439.

Antonini-Canterin, F., Pepi, M., Monte, I., Trocino, G., Barbieri, A., Barchitta, A., Ciampi, Q., Cresti, A., Miceli, S., Petrella, L. and Benedetto, F. (2020) Document addressed to cardiovascular echography operators at the time of COVID-19: A Document by the "Società Italiana di Ecocardiografia e CardioVascular Imaging" Board 2019-2021. Journal of Cardiovascular Echography 30, 2-4.

Belhadjer, Z., Méot, M., Bajolle, F., Khraiche, D., Legendre, A., Abakka, S., Auriau, J., Grimaud, M., Oualha, M., Beghetti, M., Wacker, J., Ovaert, C., Hascoet, S., Selegny, M., Malekzadeh-Milani, S., Maltret, A., Bosser, G., Giroux, N., Bonnemains, L., Bordet, J., Di Filippo, S., Mauran, P., Falcon-Eicher, S., Thambo, J.-B., Lefort, B., Moceri, P., Houyel, L., Renolleau, S. and Bonnet, D. (2020) Acute heart failure in multisystem inflammatory syndrome in children (MIS-C) in the context of global SARS-CoV-2 pandemic. Circulation (in press).

British Society of Echocardiography (2020) Clinical guidance regarding provision of echocardiography during the COVID-19 pandemic. Available at: https://bsecho.org/covid19 (Accessed: 2 April 2020)

Cameli, M., Pastore M. C., Soliman-Aboumarie, H., Mandoli, G. E., D'Ascenzi, F., Focardi, M., Cameli, P., Bigio, E., Franchi, F., Mondillo, S. and Valente, S. (2020) Usefulness of echocardiography to detect cardiac involvement in COVID-19 patients. Echocardiography (in press)

Cameli, M., Pastore, M. C., De Carli, G., Henein, M. Y., Mandoli, G. E., Lisi, E., Cameli, P., Lunghetti, S., D’Ascenzi, F., Nannelli, C., Rizzo, L., Valente, S. and Mondillo, S. (2019) ACUTE HF score, a multiparametric prognostic tool for acute heart failure: A real-life study. International Journal of Cardiology 296, 103-108. 
Cameli, M., Righini, F. M., Lisi, M., Bennati, E., Navarri, R., Lunghetti, S., Padeletti, M., Cameli, P., Tsioulpas, C., Bernazzali, S., Maccherini, M., Sani, G., Henein, M. and Mondillo, S. (2013) Comparison of right versus left ventricular strain analysis as a predictor of outcome in patients with systolic heart failure referred for heart transplantation. The American Journal of Cardiology, 112, 1778-1784.

Chambers, J. B., Garbi, M., Nieman, K., Myerson, S., Pierard, L. A., Habib, G., Zamorano, J. L., Edvardsen, T., Lancellotti, P., Delgado, V., Cosyns, B., Donal, E., Dulgheru, R., Galderisi, M., Lombardi, M., Muraru, D., Kauffmann, P., Cardim, N., Haugaa, K. and Rosenhek, R. (2017) Appropriateness criteria for the use of cardiovascular imaging in heart valve disease in adults: a European Association of Cardiovascular Imaging report of literature review and current practice. European Heart Journal - Cardiovascular Imaging 18, 489-498.

Chen, C., Chen, C., Yan, J. T., Zhou, N., Zhao, J. P. and Wang, D. W. (2020) Analysis of myocardial injury in patients with COVID-19 and association between concomitant cardiovascular diseases and severity of COVID-19. Zhonghua Xin Xue Guan Bing Za Zhi 48, E008-E008. (In Chinese)

Conticini, E., Bargagli, E., Bardelli, M., Rana, G. D., Baldi, C., Cameli, P., Gentileschi, S., Bennett, D., Falsetti, P., Lanzarone, N., Bellisai, F., Barreca, C., D'Alessandro, R., Cantarini, L. and Frediani, B. (2020) COVID-19 pneumonia in a large cohort of patients treated with biological and targeted synthetic antirheumatic drugs. Annals of the Rheumatic Diseases (in press).

Driggin, E., Madhavan, M. V., Bikdeli, B., Chuich, T., Laracy, J., BiondiZoccai, G., Brown, T. S., Der Nigoghossian, C., Zidar, D. A., Haythe, J., Brodie, D., Beckman, J. A., Kirtane, A. J., Stone, G. W., Krumholz, H. M. and Parikh, S. A. (2020) Cardiovascular Considerations for Patients, Health Care Workers, and Health Systems During the COVID19 Pandemic. Journal of the American College of Cardiology 75, 23522371.

Franchi, F., Faltoni, A., Cameli, M., Muzzi, L., Lisi, M., Cubattoli, L., Cecchini, S., Mondillo, S., Biagioli, B., Taccone, F. S. and Scolletta, S. (2013) Influence of positive end-expiratory pressure on myocardial strain assessed by speckle tracking echocardiography in mechanically ventilated patients. BioMed research international 2013, 918548918548 .

Gargani, L., Soliman-Aboumarie, H., Volpicelli, G., Corradi, F., Pastore, M. C. and Cameli, M. (2020) Why, when, and how to use lung ultrasound during the COVID-19 pandemic: enthusiasm and caution. European Heart Journal - Cardiovascular Imaging (in press).

Huang, C., Wang, Y., Li, X., Ren, L., Zhao, J., Hu, Y., Zhang, L., Fan, G., Xu, J., Gu, X., Cheng, Z., Yu, T., Xia, J., Wei, Y., Wu, W., Xie, X., Yin, W., Li, H., Liu, M., Xiao, Y., Gao, H., Guo, L., Xie, J., Wang, G., Jiang, R., Gao, Z., Jin, Q., Wang, J. and Cao, B. (2020) Clinical features of patients infected with 2019 novel coronavirus in Wuhan, China. Lancet (London, England) 395, 497-506.

Kampf, G., Todt, D., Pfaender, S. and Steinmann, E. (2020) Persistence of coronaviruses on inanimate surfaces and their inactivation with biocidal agents. The Journal of Hospital Infection 104, 246-251.

Kirkpatrick, J. N., Mitchell, C., Taub, C., Kort, S., Hung, J. and Swaminathan, M. (2020) ASE Statement on Protection of Patients and Echocardiography Service Providers During the 2019 Novel Coronavirus Outbreak: Endorsed by the American College of Cardiology. Journal of the American Society of Echocardiography: Official Publication of the American Society of Echocardiography 33, 648-653.

Lancellotti, P., Price, S., Edvardsen, T., Cosyns, B., Neskovic, A. N., Dulgheru, R., Flachskampf, F. A., Hassager, C., Pasquet, A., Gargani, L., Galderisi, M., Cardim, N., Haugaa, K. H., Ancion, A., Zamorano, J.-L., Donal, E., Bueno, H. and Habib, G. (2015) The use of echocardiography in acute cardiovascular care: Recommendations of the European Association of Cardiovascular Imaging and the Acute Cardiovascular Care Association. European Heart Journal: Acute Cardiovascular Care 4, 3-5.

Lippi, G., Lavie, C. J. and Sanchis-Gomar, F. (2020) Cardiac troponin I in patients with coronavirus disease 2019 (COVID-19): Evidence from a meta-analysis. Progress in Cardiovascular Diseases (in press).

Lisi, M., Cameli, M., Mondillo, S., Luzzi, L., Zacà, V., Cameli, P., Gotti,
G. and Galderisi, M. (2012) Incremental value of pocket-sized imaging device for bedside diagnosis of unilateral pleural effusions and ultrasound-guided thoracentesis. Interactive Cardiovascular and Thoracic Surgery 15, 596-601.

McCrindle, B. W. and Cifra, B. (2017) The role of echocardiography in Kawasaki disease. International Journal of Rheumatic Diseases 21, 5055.

Neskovic, A. N., Skinner, H., Price, S., Via, G., De Hert, S., Stankovic, I., Galderisi, M., Donal, E., Muraru, D., Sloth, E., Gargani, L., Cardim, N., Stefanidis, A., Cameli, M., Habib, G., Cosyns, B., Lancellotti, P., Edvardsen, T. and Popescu, B. A. (2018) Focus cardiac ultrasound core curriculum and core syllabus of the European Association of Cardiovascular Imaging. European Heart Journal - Cardiovascular Imaging 19, 475-481.

Noto, N., Kamiyama, H., Karasawa, K., Ayusawa, M., Sumitomo, N. and Okada, T. (2013) Long-term prognostic impact of dobutamine stress echocardiography in patients with Kawasaki disease and coronary artery lesions: a 15-year follow-up study. Journal of the American College of Cardiology 63, 337-344.

Pastore, M. C., Mandoli, G. E., Aboumarie, H. S., Santoro, C., Bandera, F., D'Andrea, A., Benfari, G., Esposito, R., Evola, V., Sorrentino, R., Cameli, P., Valente, S., Mondillo, S., Galderisi, M. and Cameli, M. (2019) Basic and advanced echocardiography in advanced heart failure: an overview. Heart Failure Reviews (in press).

Riphagen, S., Gomez, X., Gonzalez-Martinez, C., Wilkinson, N. and Theocharis, P. (2020) Hyperinflammatory shock in children during COVID-19 pandemic. Lancet (London, England) 395, 1607-1608.

Sciaccaluga, C., D'Ascenzi, F., Mandoli, G. E., Rizzo, L., Sisti, N., Carrucola, C., Cameli, P., Bigio, E., Mondillo, S. and Cameli, M. (2020) Traditional and novel imaging of right ventricular function in patients with heart failure and reduced ejection fraction. Current heart failure reports 17, 28-33.

Sinha, I. P., Harwood, R., Semple, M. G., Hawcutt, D. B., Thursfield, R., Narayan, O., Kenny, S. E., Viner, R., Hewer, S. L. and Southern, K. W. (2020) COVID-19 infection in children. The Lancet Respiratory Medicine 8, 446-447.

Skulstad, H., Cosyns, B., Popescu, B. A., Galderisi, M., Salvo, G. D., Donal, E., Petersen, S., Gimelli, A., Haugaa, K. H., Muraru, D., Almeida, A. G., Schulz-Menger, J., Dweck, M. R., Pontone, G., Sade, L. E., Gerber, B., Maurovich-Horvat, P., Bharucha, T., Cameli, M., Magne, J., Westwood, M., Maurer, G. and Edvardsen, T. (2020) COVID-19 pandemic and cardiac imaging: EACVI recommendations on precautions, indications, prioritization, and protection for patients and healthcare personnel. European Heart Journal Cardiovascular Imaging 21, 592-598.

Sosa, T., Brower, L. and Divanovic, A. (2019) Diagnosis and management of kawasaki disease. JAMA Pediatrics 173, 278.

Tsuda, E. and Singhal, M. (2017) Role of imaging studies in Kawasaki disease. International Journal of Rheumatic Diseases 21, 56-63.

Valente, S., Anselmi, F. and Cameli, M. (2020) Acute coronary syndromes during COVID-19. European Heart Journal 41, 2047-2049.

van Doremalen, N., Bushmaker, T., Morris, D. H., Holbrook, M. G., Gamble, A., Williamson, B. N., Tamin, A., Harcourt, J. L., Thornburg, N. J., Gerber, S. I., Lloyd-Smith, J. O., de Wit, E. and Munster, V. J. (2020) Aerosol and surface stability of $\mathrm{HCoV}-19$ (SARS-CoV-2) compared to SARS-CoV-1. medRxiv (in press).

Verdoni, L., Mazza, A., Gervasoni, A., Martelli, L., Ruggeri, M., Ciuffreda, M., Bonanomi, E. and D'Antiga, L. (2020) An outbreak of severe Kawasaki-like disease at the Italian epicentre of the SARS-CoV-2 epidemic: an observational cohort study. Lancet (London, England) 395, 1771-1778.

World Health Organization (2020a) Rational use of personal protective equipment for coronavirus disease (COVID19): interim guidance, 27 February 2020. Available at: https://apps.who.int/iris/bitstream/handle/10665/331215/ WHO-2019-nCov-IPCPPE_use-2020.1-eng.pdf; (Accessed: 27 February 2020). 
World Health Organization (2020b) Rational use of personal protective equipment (PPE) for coronavirus disease (COVID-19): interim guidance, 19 March 2020. Available at: https://apps.who.int/iris/ bitstream/handle/10665/331498/WHO-2019-nCoV-IPCPP E_use-2020.2-eng.pdf?sequence=1\&isAllowed=y (Accessed:
19 March 2020)

Wu, Z. and McGoogan, J. M. (2020) Characteristics of and Important Lessons From the Coronavirus Disease 2019 (COVID-19) Outbreak in China. JAMA 323, 1239. 